\title{
Mediastinal Melanotic Schwannoma
}

National Cancer Institute

\section{Source}

National Cancer Institute. Mediastinal Melanotic Schwannoma. NCI Thesaurus. Code C6635.

A melanotic schwannoma that affects the mediastinum. 